\title{
Within-Session Analysis of Amphetamine-Elicited Rotation Behavior Reveals Differences between Young Adult and Middle- Aged F344/BN Rats with Partial Unilateral Striatal Dopamine Depletion
}

\author{
Crystal S. Bethel-Brown ${ }^{1}$, Hongyu Zhang ${ }^{1}$, Stephen C. Fowler ${ }^{4,5,6}$, Mark E. Chertoff ${ }^{2,4}$, G. \\ Stennis Watson ${ }^{7}$, and John A. Stanford ${ }^{1,3,4}$ \\ ${ }^{1}$ Department of Molecular \& Integrative Physiology, University of Kansas Medical Center, Kansas \\ City, KS 66160 \\ ${ }^{2}$ Department of Hearing \& Speech, University of Kansas Medical Center, Kansas City, KS 66160 \\ ${ }^{3}$ Landon Center on Aging, University of Kansas Medical Center, Kansas City, KS 66160 \\ ${ }^{4}$ Kansas Intellectual \& Developmental Disabilities Research Center, University of Kansas Medical \\ Center, Kansas City, KS 66160 \\ ${ }^{5}$ Department of Pharmacology \& Toxicology, University of Kansas, Lawrence, KS 66045 \\ ${ }^{6}$ Schiefelbusch Institute for Life Span Studies, University of Kansas, Lawrence, KS 66045 \\ ${ }^{7}$ Department of Psychiatry and Behavioral Sciences, University of Washington School of \\ Medicine and the Geriatric Research, Education, and Clinical Center (GRECC), VA Puget Sound \\ Health Care System, Seattle, WA 98108
}

\begin{abstract}
Preclinical modeling of Parkinson's disease using 6-hydroxydopamine (6-OHDA) has been valuable in developing and testing therapeutic strategies. Recent efforts have focused on modeling early stages of disease by infusing 6-OHDA into the striatum. The partial DA depletion that follows intrastriatal 6-OHDA is more variable than the near complete depletion following medial forebrain bundle infusion, and behavioral screening assays are not as well characterized in the partial lesion model. We compared relationships between amphetamine-elicited rotation behavior and DA depletion following intrastriatal 6-OHDA $(12.5 \mu \mathrm{g})$ in 6 month vs. 18 month F344/BN rats, at 2-weeks and 6-weeks post-lesion. We compared the total number of rotations with withinsession (bin-by-bin) parameters of rotation behavior as indicators of DA depletion. Striatal DA depletion was greater in the young adult than in the middle-aged rats at 2 weeks but not at 6 weeks post-lesion. The total number of rotations for the whole session and striatal DA depletion did not differ between the two age groups. Regression analysis revealed a greater relationship between within-session parameters of rotation behavior and DA depletion in the middle-aged group than in the young adult group. These results have implications for estimating DA depletion in preclinical studies using rats of different ages.
\end{abstract}

\section{(C) 2010 Elsevier Inc. All rights reserved}

*Correspondence to: John A. Stanford, Ph.D. Department of Physiology, Mailstop 3051 University of Kansas Medical Center 3901 Rainbow Blvd., Kansas City, KS 66160 phone: 913-588-7416 jstanford@ kumc.edu.

Publisher's Disclaimer: This is a PDF file of an unedited manuscript that has been accepted for publication. As a service to our customers we are providing this early version of the manuscript. The manuscript will undergo copyediting, typesetting, and review of the resulting proof before it is published in its final citable form. Please note that during the production process errors may be discovered which could affect the content, and all legal disclaimers that apply to the journal pertain. 


\section{Keywords}

Parkinson's disease; animal models; behavioral assay; basal ganglia; rotation; striatum; amphetamine

\section{Introduction}

Preclinical modeling of Parkinson's disease (PD) using neurotoxins such as 6hydroxydopamine (6-OHDA) has been essential to developing and testing potential therapeutic strategies. The most common protocol involves a unilateral infusion of 6-OHDA into the medial forebrain bundle in rats. This method produces a near complete dopamine (DA) depletion in the ipsilateral striatum, a substantial decrease in the number of DA neurons in the substantia nigra, and severe deficits in contralateral limb use (reviewed by Deumans et al., 2002). These effects are analogous to those that accompany advanced PD. Because of the dopaminergic imbalance between the lesioned and the non-lesioned hemispheres, rats exhibit rotational behavior following the systemic administration of DA agonists (Hudson et al., 1993; Ungerstedt \& Arbuthnott, 1970). The fact that apomorphineand amphetamine-elicited rotations are effective behavioral screens for near-complete (i.e., $>90 \%$ ) unilateral DA depletions (Hudson et al., 1993) make these assays widely used in preclinical PD studies.

Due to increased interest in neuroprotective treatment strategies during initial stages of degeneration, recent efforts have focused on developing sub-maximal but progressive DA depletions with 6-OHDA in rats (e.g., Barneoud et al., 2000; Deumens et al., 2002; Fleming et al., 2005; Kirik et al., 1998; Yuan et al., 2005). The goal of these studies is to model early stages of the disease. One difficulty inherent in such preclinical studies is estimating the degree of DA depletion prior to post mortem quantification. Although rotation assays reliably predict advanced striatal DA depletions, their predictive utility in partial lesion studies is less clear. Several research groups have incorporated sensitive motor assessment techniques, such as schedule-controlled operant behavior (Lindner et al., 1999), evaluation of forelimb asymmetry (Fleming et al., 2005), and tests of skilled paw use and postural adjustments (Barneoud et al., 2000) in their early-stage PD models. Despite the fact that these tests have great clinical relevance, their implementation can be laborious, sometimes involve direct investigator involvement, or may be unfeasible for large scale studies. For these reasons, rotation-based assays remain the most widely-used behavioral screens in preclinical PD studies. Continued characterization and optimization for studies involving early-stage models is therefore warranted.

A potential weakness of many preclinical PD studies is the exclusive use of young adult instead of older animals to model this age-related disease. This is especially relevant to partial lesion studies, as there are well-documented compensatory processes that accompany the early stages of idiopathic and experimental PD (Zigmond, 1997), and many of these compensatory processes may be affected by normal aging (Gould et al., 1996; Stanford et al., 2001; Yurek \& Fletcher-Turner, 2001). While a few studies have addressed this issue by testing 6-OHDA in older animals (e.g., Cass et al., 2002; Lindner et al., 1999), to our knowledge there are no published studies comparing conventional measures of drug-elicited rotation behavior between unilaterally-lesioned young and middle-aged animals. The purpose of the current study was to characterize the effects of an intrastriatal 6-OHDA lesion model of early-stage PD in young adult versus middle-aged rats. Our first aim was to determine the relationship between amphetamine-elicited rotation behavior and the degree of striatal DA depletion in this partial lesion model. Although apomorphine is also used to elicit rotations in the unilateral 6-OHDA model, we limited our tests to amphetamine 
because of its greater sensitivity in tests involving partial DA depletions (Hefti et al., 1980; Hudson et al., 1993). Our second aim was to determine whether the relationship between amphetamine-elicited rotation behavior and DA depletion differed between the two age groups.

\section{Materials and Methods}

\section{Animals}

Thirty-four F344/BN rats ( $\mathrm{n}=18$ six-month-old; $\mathrm{n}=16$ eighteen-month-old; ages are at the time of lesion) were obtained from NIA colonies. Animals were housed 2 per cage in the University of Kansas Medical Center's (KUMC's) AAALAC accredited animal facility, and were maintained on ad libitum food and water, and a 12 hour lights on/off cycle (lights on from 8:00 am to 8:00 pm). Animals were tested during the light phase of the cycle.

Procedures were approved by the KUMC IACUC and adhered to the Guide for the Care and Use of Laboratory Animals (National Research Council, 1996).

\section{Unilateral Intrastriatal 6-OHDA Infusion}

Animals were anesthetized with sodium pentobarbital $(40 \mathrm{mg} / \mathrm{kg})$, and placed in a stereotaxic frame. A burr hole was placed in the skull over the right striatum (AP $+1.0 \mathrm{~mm}$, $\mathrm{ML}+3.5 \mathrm{~mm}$ vs. bregma). A 26 gauge dome tipped needle connected to a microliter syringe via Teflon tubing was lowered into the striatum $(5.0 \mathrm{~mm}$ from the dural surface). In the lesion groups, $12.5 \mu \mathrm{g}$ 6-OHDA $(5.0 \mu \mathrm{l}$, at $2.5 \mu \mathrm{g} / \mu \mathrm{l}$, in $0.9 \%$ saline with $0.1 \%$ ascorbic acid) was infused at a rate of $0.25 \mu \mathrm{L} / \mathrm{min}$. The dose of 6-OHDA was based on previous studies examining the effects of intrastriatal 6-OHDA (Cousins \& Salamone, 1996), and the volume was based on our work examining the effects of intrastriatal delivery of glial cell line-derived neurotrophic factor on nigrostriatal function (Salvatore et al., 2009; Stanford et al., 2007). Following an additional 5 min the needle was slowly withdrawn. Bone wax was applied, the wound was closed with wound clips, and animals were allowed to recover in their home cages.

\section{Amphetamine-Elicited Rotation}

Two weeks after the lesions, all animals were tested for amphetamine-elicited rotation behavior. Animals were injected with d-amphetamine sulfate $(5 \mathrm{mg} / \mathrm{kg}$, s.c.) and placed in a rotometer for 60 minutes. The rotometer was a $26.5 \mathrm{~cm}$ diameter cylinder placed atop a force-sensing actometer surface (see Fowler et al., 2001). Custom software quantified the number of ipsiversive rotations (i.e., toward the lesioned side) in untethered animals as a function of 3-min time bins. On the day following behavioral testing, brains were removed from the 2-week group for analysis of dopamine content. The 6-week group was kept for four more weeks so that amphetamine-elicited rotation could be tested again at 6 weeks post-lesion. Like the 2-week group, their brains were removed on the day following behavioral testing.

\section{Whole Tissue Levels of DA \& DOPAC}

On the day following behavioral testing, rats' brains were dissected for fresh tissue harvest. Brains were placed in a chilled brain mold and $1 \mathrm{~mm}$ sections were made allowing free-hand dissection of bilateral dorsal striatum. Striatal tissue was processed and analyzed for levels of DA and dihydroxyphenylacetic acid (DOPAC; the primary DA metabolite in rats) as described previously (Enna et al., 2006). Briefly, levels of DA and DOPAC were quantified using an isocratic high pressure liquid chromatography system coupled to a dual-channel electrochemical array detector (ESA Inc., Chelmsford, MA; Model 5100A, $\mathrm{E}_{1}+0.35 \mathrm{mV}$ and $\mathrm{E}_{2}-0.25 \mathrm{mV}$ using a 5011 dual analytical cell). 


\section{Data Analysis}

We used two methods to quantify relationships between rotation behavior and striatal DA depletion (expressed as the percentage of DA in the lesioned striatum versus the contralateral striatum). These data were calculated separately for the young and middle-aged rats, and all animals were included in analyses regardless of their rotation behavior. First we calculated correlation coefficients between the total number of rotations per 60-min session and the percentage of DA depletion across both post-lesion time points. We then examined rotation behavior in more detail by plotting the number of rotations by 3-min time bins. This within-session "kinetic" analysis revealed a sigmoidal function, so we used GraphPad Prism 4 (GraphPad Software Inc., San Diego, CA) to fit each animal's rotation data to the following sigmoidal curve: $\mathrm{Y}=\mathrm{Bottom}+(\mathrm{Top}-\mathrm{Bottom}) /(1+\exp ((\mathrm{T} 50-\mathrm{X}) /$ Slope $))$, where Bottom $=$ zero rotations, $\mathrm{Top}=$ maximum rotations (so $\mathrm{Y}=$ the total number of rotations during the session), $\mathrm{T} 50=$ time bin in which the number of rotations was halfway between zero and $\mathrm{Y}$, and Slope $=$ steepness of the curve. Within-session rotation behavior fit this curve well, with a mean $\mathrm{R}^{2}$ across all animals of $0.91 \pm 0.04$. We conducted the following exploratory analyses to determine whether these best fit values (T50, Slope, and $\mathrm{R}^{2}$ [how well each curve fit the function]) were related to DA depletion over and above the association between DA depletion and the total number of rotations (PASW Statistics version 18, SPSS, Inc. Chicago, IL). First, zero order Pearson correlation coefficients were computed for DA depletion by T50, Slope, and $\mathrm{R}^{2}$ for each age group. Second, best fit variables with significant correlations were entered into a two-step hierarchical multiple regression model using forced entry of independent variables: Step 1, DA depletion was regressed on total number of rotations; Step 2, DA depletion was regressed on the combination of significant best fit variables and the total number of rotations. Third, we examined the resulting models for overall significance, the change in variance explained by the second model $\left(\Delta \mathrm{R}^{2}\right)$, standardized regression weights, and partial correlations for variables in the second model.

\section{Results}

\section{A. Dopamine \& Total Number of Rotations}

Whole tissue levels of DA and DOPAC in the lesioned and contralateral striatum are presented in Table 1. Dopamine depletion in the lesioned striatum (expressed as \% of the nonlesioned side) was greater in the young group than in the middle-aged group at two weeks, $t(1,14)=4.700, p<0.05$, but not at 6 weeks post-lesion (Figure 1a). The total number of rotations for the whole session, which did not differ significantly between the two age groups at either time after lesion (Figure 1b), correlated modestly but significantly with the percentage of DA depletion for both the young $(\mathrm{r}=0.49, \mathrm{p}<0.05)$ and the middle-aged $(\mathrm{r}=0.59, \mathrm{p}<0.05)$ rats.

\section{B. Within-Session Rotation Kinetics}

When we examined rotation behavior in more detail by plotting the number of rotations by 3-min time bins, a sigmoidal function was revealed (Figure 2). This function allowed for the analysis of within-session "kinetic" variables of rotation behavior, including T50 (time bin in which the number of rotations was halfway between zero and maximum). For older animals, the association between DA depletion and T50 was significant $(\mathrm{r}=0.672, \mathrm{p}=$ $0.003)$. Otherwise, we did not observe significant correlations between DA depletion and goodness of fit variables for younger or older animals ( $p$-values $>0.10$ ). As a result, we conducted hierarchical regression analysis only for older animals, with DA depletion as the dependent variable and the total number of rotations alone as the independent variable on step 1 (Model 1), and both total number of rotations and T50 as the independent variables on step 2 (Model 2). Both models were significant: Model 1 (DA depletion $=$ total number of 
rotations), $\mathrm{R}^{2}=0.352, \mathrm{~F}(1,14)=7.068, \mathrm{p}=0.020$ and Model $2(\mathrm{DA}$ depletion $=$ total number of rotations, T50), $\mathrm{R}^{2}=0.639, \mathrm{~F}(2,13)=10.636, \mathrm{p}=0.009$. Furthermore, Model 2 accounted for significantly more variance than did Model $1, \Delta \mathrm{R}^{2}=0.287, \mathrm{~F}(1,12)=9.554, \mathrm{p}$ $=0.009$. Regression coefficients are positive (Table 2), showing that increasing DA depletion is associated with increasing number of rotations $(\beta=0.593)$ in Model 1 or increasing number of rotations $(\beta=0.449)$ and increasing T50 $(\beta=0.555)$ in Model 2. (For comparison, we repeated this set of analyses for younger animals, but neither $R^{2}$ nor $\Delta R^{2}$ was significant for the addition of T50, both p-values $>0.10$.) Regression coefficients (Table $2 \mathrm{~b}$ ) were used to calculate "predicted DA depletion" from the total number of rotations and T50 values (Figure 3). Taken together, these results demonstrate that the number of amphetamine-induced rotations is a significant predictor of 6-OHDA-induced striatal DA depletion, and that predictive power is further significantly increased when time to complete $50 \%$ of the total rotations is considered in middle-aged rats.

\section{Discussion}

We report here age-related differences in relationships between unilateral striatal DA depletion and within-session kinetic characteristics of amphetamine-elicited rotation behavior in F344/BN rats. The DA depleting effect of intrastriatal 6-OHDA was attenuated in the middle-aged rats at two weeks post-lesion. DA depletion did not differ between the two age groups at the 6 weeks time point. The correlation between the number of amphetamine-elicited rotations and DA depletion was significant for both age groups. Notably, the addition of T50 to the regression model significantly added to the amount of variance in DA depletion explained by the total number of rotations alone in older (but not younger) animals. These results suggest that in studies involving older animals, withinsession analyses of drug-elicited rotation behavior may provide a better indication of percent DA depletion in a partial lesion model than the total number of rotations.

There is increased interest in developing neuroprotective therapeutic strategies for use in initial stages of degeneration in PD and other neurodegenerative disorders. The intrastriatal 6-OHDA protocol is considered to be a valid model of early-stage PD (Bjorkland et al., 1997; Blandini et al., 2007). The fact that within-session kinetic parameters of amphetamine-elicited rotation behavior were more correlated with the degree of DA depletion in middle-aged rats in this partial lesion model has significant implications for preclinical PD research. Although strong rotation behavior can reliably predict advanced DA depletions (Hudson et al., 1993), estimating partial depletions is a more elusive goal. Previous studies have demonstrated that rats with partial unilateral DA loss rotate in response to amphetamine (Hefti et al., 1980; Hudson et al., 1993; Barneoud et al., 2000; Yuan et al., 2005). However, measures of total rotations or rotation rate were at best able to differentiate between partial and maximal DA depletions. Although tests of paw reaching ability provide valuable preclinical measures of bradykinesia and manual dexterity that correlate with a wider range (40-99\%) of DA depletion (Barneoud et al., 1995; Whishaw et al., 1997), drug-elicited rotation behavior is a more widely-used assay that is easier to implement. Our results suggest that while the total number of amphetamine-elicited rotations moderately (but significantly) reflects striatal DA depletion levels in young rats, a bin-by-bin analysis of rotation behavior greatly enhances the utility of this assay in middleaged rats.

It is unclear why within-session rotation parameters correlated greater with DA depletion in the middle-aged rats than in the young rats. Compensatory processes, such as increased DA release in residual nigrostriatal terminals (Zigmond et al., 1984) and normalization of extracellular DA content (Bergstrom \& Garris, 2003) have been reported following 6OHDA depletion in rats. It is possible that age-related differences in these processes 
(Stanford et al., 2001) altered the relationship between amphetamine-elicited rotation behavior and DA depletion. The onset of many age-related changes in nigrostriatal function occurs in middle-age in these animals (e.g., Hebert \& Gerhardt, 1998; Yurek et al., 1998), so it may be that greater variability in this group contributed to this effect. The greater variability in rotation behavior and DA depletion in the middle-aged rats supports this hypothesis. Given the increased variability in the middle-aged group, it could be argued that the greater correlation was more statistical artifact than physiological effect. However, the difference between the age groups in the regression correlations $\left(R^{2}=0.19\right.$ vs 0.63$)$ versus the simple correlations for total number of rotations and DA depletion $\left(R^{2}=0.24\right.$ vs 0.34$)$ suggest otherwise.

Age-related changes in the psychomotor stimulating effects of amphetamine may have also played a role in our findings. In fact, at two weeks post-lesion, within-session onset of rotations occurred earlier in the younger group, and young rats reached asymptotic rotation levels within the 60 minute session, while middle-aged rats did not. Because T50 was not a significant contributor of variance in the regression model for the young adult group, the greater DA depletion in this group at this time point likely does not underlie this difference. Previous studies have reported age-related decreases in the number and function of DA transporters (Hebert et al., 1999) as well as in amphetamine-stimulated DA release (Yurek et al., 1998). It is therefore possible that amphetamine's effects were greater in young animals. Our previously-reported findings that amphetamine affects striatal electrophysiological activity differently in freely-moving aged rats compared to young adult rats (Stanford \& Gerhardt, 2001; Stanford et al., 2002) are consistent with this hypothesis. The fact that rotation behavior reached asymptote in the young adult within the 60-minute session while it did not in the middle-aged rats may be related to changes in amphetamine pharmacokinetics with aging (Truex \& Schmidt, 1980).

Our finding regarding the earlier within-session onset of rotations at the 2 week time point compared to the 6 week time point in the young adult group is conspicuous. One limitation of our study is that we did not quantify DA in the substantia nigra, where amphetamine produces much of its locomotor effect (Jackson \& Kelly, 1983). It is possible that this difference may reflect greater nigral involvement in this neurobehavioral relationship. Retrograde DA depletion produced by intrastriatal 6-OHDA involves early oxidative damage of DA terminals followed by a cascade of events that culminates in nigral cell death (Blandini et al., 2007; Sanchez-Iglesias et al., 2007; Sauer \& Oertel, 1994). Although amphetamine-elicited rotation behavior is sensitive to early loss of DA in the striatum, rotation parameters may be more intimately related to the integrity of the nigrostriatal and the striatonigral pathways. Previous studies reporting significant relationships between DA agonist-elicited rotation behavior and nigral DA loss (Hudson et al., 1993; Olds et al., 2006; Robertson \& Robertson, 1989) support this hypothesis. Striatonigral neurons synapse in the substantia nigra reticulata, forming the so-called "direct pathway" through which the basal ganglia projects to the motor thalamus and facilitates movement (Bolam et al, 2000). The substantia nigra reticulata also receives DA input from the substantia nigra compacta and the nucleus accumbens (reviewed by Hauber, 1998). Amphetamine increases DA activity both of these nuclei (Jackson \& Kelly, 1983; Sharp et al., 1987), and this extrastriatal DA release may have influenced rotation behavior differently at the earlier time point. Clearly, further studies are required to determine relationships between motor function, initial striatal DA loss, and eventual nigrostriatal DA depletion.

Our finding that DA depletion was greater in young rats at two weeks post-lesion than in middle-aged rats was somewhat unexpected. Previous studies have reported enhanced DA depletion in older rats following intracerebroventricular or midbrain delivery of 6-OHDA (Cass et al., 2002; Marshall et al., 1983). Although we did not measure this directly, it is 
possible that age-related decreases in both the number and function of DA transporters resulted in diminished uptake of 6-OHDA into striatal DA terminals. Significant decreases in striatal DA transporter binding sites occur in F344 rats beginning at 18 months of age (Hebert et al., 1999), and the uptake capacity of DA transporter sites is significantly diminished in F344 rats beginning at 12 months (Hebert and Gerhardt, 1999). This may explain previous reports that at 12 weeks post-lesion, intrastriatal 6-OHDA resulted in similar nigral DA cell loss and striatal DA depletion in young versus middle-aged rats (Lindner et al., 1999). These findings suggest that 6-OHDA infusion site and post-lesion duration are important factors in preclinical modeling of PD.

Overall, our results indicate that matching animals according to measures that reflect the within-session onset and bin-by-bin trajectory of amphetamine-elicited rotation behavior may be a more effective strategy for estimated DA depletion prior to verification following tissue harvest. This method would be of value when equating drug and vehicle groups prior to therapeutic intervention in middle-aged animals. Further studies validating this method using a greater number of rats are warranted to test this hypothesis.

\section{Acknowledgments}

This study was supported by NIH grants AG023549 (JAS), AG026491 (JAS), MH43429 (SCF), AG23640 (GSW), the Smith Intellectual and Developmental Disabilities Research Center (HD02528), and a Lied Endowed Basic Science Research Grant (JAS).

\section{References}

Barneoud P, Descombris E, Aubin N, Aubrous DN. Evaluation of simple and complex sensorimotor behaviours in rats with a partial lesion of the dopaminergic nigrostriatal system. Eur. J. Neurosci. 2000; 12:322-336. [PubMed: 10651887]

Bergstrom BP, Garris PA. 'Passive stabilization' of striatal extracellular dopamine across the lesion spectrum encompassing the presymptomatic voltammetric study in the 6-OHDA-lesioned rat. J. Neurochem. 2003; 87:1224-1236. [PubMed: 14622102]

Bjorklund A, Rosenblad C, Winkler C, Kirik D. Studies on neuroprotective and regenerative effects of GDNF in a partial lesion model of Parkinson's disease. Neurobiol Dis. 1997; 4:186-200. [PubMed: 9361295]

Blandini F, Levandis G, Bazzini E, Nappi G, Armentero MT. Time-course of nigrostriatal damage, basal ganglia metabolic changes and behavioural alterations following intrastriatal injection of 6hydroxydopamine in the rat: new clues from an old model. Eur J Neurosci. 2007; 25:397-405. [PubMed: 17284180]

Bolam JP, Hanley JJ, Booth PAC, Bevan MD. Synaptic organisation of the basal ganglia. J Anat. 2000; 196:527-542. [PubMed: 10923985]

Cass WA, Harned ME, Bailey SL. Enhanced effects of 6-hydroxydopamine on evoked overflow of striatal dopamine in aged rats. Brain Res. 2002; 938:29-37. [PubMed: 12031532]

Cousins MS, Salamone JD. Involvement of ventrolateral striatal dopamine in movement initiation and execution: A microdialysis and behavioral investigation. Neuroscience. 1996; 70:849-859. [PubMed: 8848171]

Deumans R, Blokland A, Prickaerts J. Modeling Parkinson's disease in rats: an evaluation of 6-OHDA lesions of the nigrostriatal pathway. Exp Neurol. 2002; 175:303-317. [PubMed: 12061862]

Enna SJ, Reisman SA, Stanford JA. CGP 56999A, a GABAB receptor antagonist, enhances expression of brain-derived neurotrophic factor and attenuates dopamine depletion in the rat corpus striatum following a 6-hydroxydopamine lesion of the nigrostriatal pathway. Neurosci Lett. 2006; 406:102106. [PubMed: 16890350]

Fleming SM, Delville Y, Schallert T. An intermittent, controlled-rate, slow progressive degeneration model of Parkinson's disease: antiparkinson effects of Sinemet and protective effects of methylphenidate. Behav Brain Res. 2005; 156:201-213. [PubMed: 15582106] 
Hauber W. Involvement of basal ganglia transmitter systems in movement initiation. Prog Neurobiol. 1998; 56:507-540. [PubMed: 9775402]

Hebert MA, Gerhardt GA. Normal and drug-induced locomotor behavior in aging: comparison to evoked DA release and tissue content in Fischer 344 rats. Brain Res. 1998; 797:42-54. [PubMed: 9630504]

Fowler SC, Birkestrand BR, Chen R, Moss SJ, Vorontsova E, Wang G, Zarcone TJ. A force-plate actometer for quantitating rodent behaviors: illustrative data on locomotion, rotation, spatial patterning, stereotypies, and tremor. J Neurosci Methods. 2001; 107:107-124. [PubMed: 11389948]

Gould TJ, Stromberg I, Bickford PC. Decline in striatal dopamine D1 and D2 receptor activation in aged F344 rats. Neurobiol Aging. 1996; 17:877-883. [PubMed: 9363799]

Hauber W. Involvement of basal ganglia transmitter systems in movement initiation. Prog Neurobiol. 1998; 56:507-540. [PubMed: 9775402]

Hebert MA, Gerhardt GA. Age-related changes in the capacity, rate, and modulation of dopamine uptake within the striatum and nucleus accumbens of Fischer 344 rats: an in vivo electrochemical study. J Pharmacol Exp Ther. 1999; 288:879-887. [PubMed: 9918602]

Hebert MA, Larson GA, Zahniser NR, Gerhardt GA. Age-related reductions in [3H]WIN 35,428 binding to the dopamine transporter in nigrostriatal and mesolimbic brain regions of the Fischer 344 rat. J Pharmacol Exp Ther. 1999; 288:1334-1339. [PubMed: 10027875]

Hefti F, Melamed E, Sahakian BJ, Wurtman RJ. Circling behavior in rats with partial, unilateral nigrostriatal lesions: effect of amphetamine, apomorphine, and L-DOPA. Pharmacol Biochem Behav. 1980; 12:185-188. [PubMed: 7189592]

Hudson JL, van Horne CG, Stromberg I, Brock S, Clayton J, Masserano J, Hoffer BJ, Gerhardt GA. Correlation of apomorphine- and amphetamine-induced turning with nigrostriatal dopamine content in unilateral 6-hydroxydopamine lesioned rats. Brain Res. 1993; 626:167-174. [PubMed: 8281427]

Jackson EA, Kelly PH. Role of nigral dopamine in amphetamine-induced locomotor activity. Brain Res. 1983; 278:366-369. [PubMed: 6640328]

Kirik D, Rosenblad C, Bjorklund A. Characterization of behavioral and neurodegenerative changes following partial lesions of the nigrostriatal dopamine system induced by intrastriatal 6hydroxydopamine in the rat. Exp Neurol. 1998; 152:259-277. [PubMed: 9710526]

Lindner MD, Cain CK, Plone MA, Frydel BR, Blaney TJ, Emerich DF, Hoane MR. Incomplete nigrostriatal dopaminergic cell loss and partial reductions in striatal dopamine produce akinesia, rigidity, tremor and cognitive deficits in middle-aged rats. Behav Brain Res. 1999; 102:1-16. [PubMed: 10403011]

Marshall JF, Drew MC, Neve KA. Recovery of function after mesotelencephalic dopaminergic injury in senescence. Brain Res. 1983; 259:249-260. [PubMed: 6297672]

Olds ME, Jacques DB, Kopyov O. Relation between rotation in the 6-OHDA lesioned rat and dopamine loss in striatal and substantia nigra subregions. Synapse. 2006; 59:532-544. [PubMed: 16565974]

Robertson GS, Robertson HA. Evidence that L-dopa-induced rotational behaviour is dependent on both striatal and nigral mechanisms. J Neurosci. 1989; 9:3326-3331. [PubMed: 2795165]

Salvatore MF, Gerhardt GA, Dayton RD, Klein RL, Stanford JA. Bilateral effects of unilateral GDNF administration on dopamine- and GABA-regulating proteins in the rat nigrostriatal system. Exp Neurol. 2009; 219:197-207. [PubMed: 19460370]

Sanchez-Iglesias S, Rey P, Mendez-Alvarez E, Labandeira-Garcia JL, Soto-Otero R. Time-course of brain oxidative damage caused by intrastriatal administration of 6-hydroxydopamine in a rat model of Parkinson's disease. Neurochem Res. 2007; 32:99-105. [PubMed: 17160721]

Sauer H, Oertel WH. Progressive degeneration of nigrostriatal dopamine neurons following intrastriatal terminal lesions with 6-hydroxydopamine: a combined retrograde tracing and immunocytochemical study in the rat. Neuroscience. 1994; 59:401-415. [PubMed: 7516500]

Sharp T, Zetterstrom T, Ljungberg T, Ungerstedt U. A direct comparison of amphetamine-induced behaviours and regional brain dopamine release in the rat using intracerebral dialysis. Brain Res. 1987; 401:322-330. [PubMed: 3815099] 
Stanford JA, Gash CR, Gerhardt GA. Aged F344 rats exhibit an increased proportion of dopamine agonist-excited striatal neurons. Neurobiol Aging. 2002; 23:263-270. [PubMed: 11804712]

Stanford JA, Gerhardt GA. Age-related changes in striatal function of freely-moving F344 rats. Neurobiol Aging. 2001; 22(4):659-669. [PubMed: 11445266]

Stanford, JA.; Hebert, MA.; Gerhardt, GA. Biochemical and anatomical changes in basal ganglia of aging animals. In: Hof, PR.; Mobbs, CV., editors. Functional Neurobiology of Aging. Academic Press; San Diego: 2001. p. 727-735.

Stanford JA, Salvatore MF, Joyce BM, Zhang H, Gash DM, Gerhardt GA. Bilateral effects of unilateral intrastriatal GDNF on locomotor-excited and nonlocomotor-related striatal neurons in aged F344 rats. Neurobiol Aging. 2007; 28:156-165. [PubMed: 16314001]

Truex LL, Schmidt MJ. 3-H-amphetamine concentrations in the brains of young and aged rats: implications for assessment of drug effects in aged animals. Neurobiol Aging. 1980; 1:93-95. [PubMed: 7196508]

Ungerstedt U, Arbuthnott GW. Quantitative recording of rotational behavior in rats after 6-hydroxydopamine lesions of the nigrostriatal dopamine system. Brain Res. 1970; 24:485-493. [PubMed: 5494536]

Yuan H, Sarre S, Ebinger G, Michotte Y. Histological, behavioural and neurochemical evaluation of medial forebrain bundle and striatal 6-OHDA lesions as rat models of Parkinson's disease. J Neurosci Meth. 2005; 144:35-45.

Yurek DM, Fletcher-Turner A. Lesion-induced increase of BDNF is greater in the striatum of young versus old rat brain. Exp Neurol. 2001; 161:392-396. [PubMed: 10683304]

Zigmond MJ. Do compensatory processes underlie the preclinical phase of neurodegenerative disease? Insights from an animal model of parkinsonism. Neurobiol Dis. 1997; 4:247-253. [PubMed: 9361301] 
A

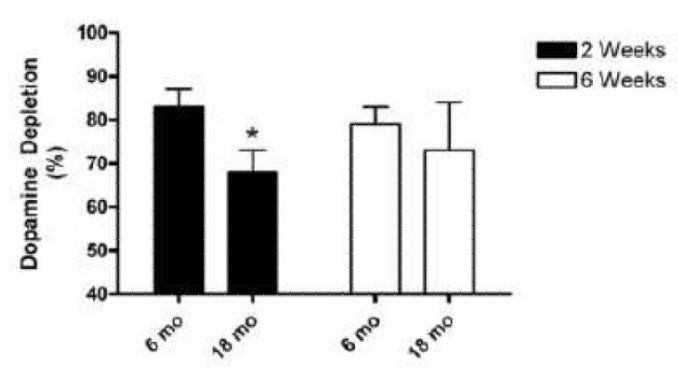

B

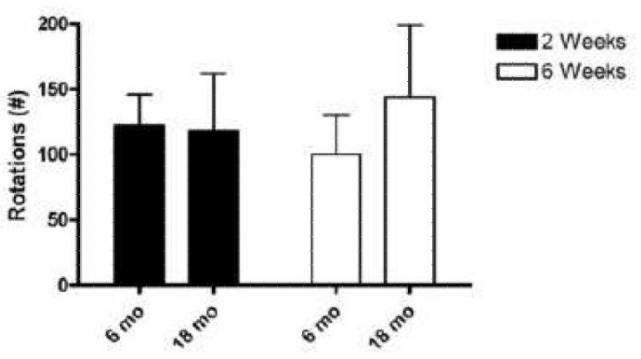

Figure 1.

Striatal dopamine depletion (A) and total number of rotations (B) in young $(6 \mathrm{mo})$ and middle-aged (18 mo) rats at 2 weeks and 6 weeks post-lesion. Dopamine depletion was significantly less in middle-aged rats than in young rats at 2 weeks, but not at 6 weeks postlesion $(* \mathrm{p}<0.05)$. The total number of rotations did not differ between the two age groups at either post-lesion timepoint. 

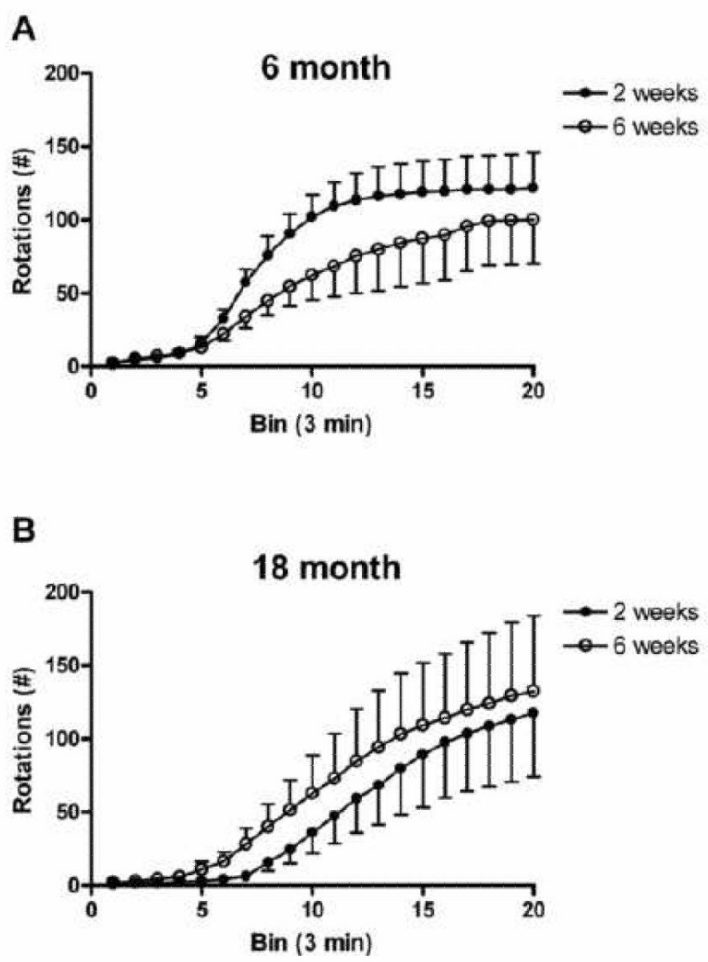

Figure 2.

Within-session analysis of rotation behavior as a function of each 3 min time bin and weeks post-lesion in (A) 6-month-old and (B) 18-month-old rats. Values within each bin represent the cumulative number of rotations at each time point. Although the total number of rotations was the same between the young and middle-aged rats, young rats exhibited an earlier and steeper onset of rotations at 2 weeks but not at 6 weeks. Error bars $=$ SEM. 


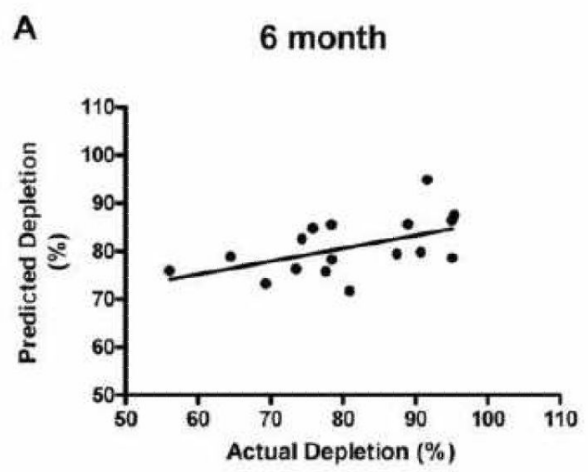

B

\section{8 month}

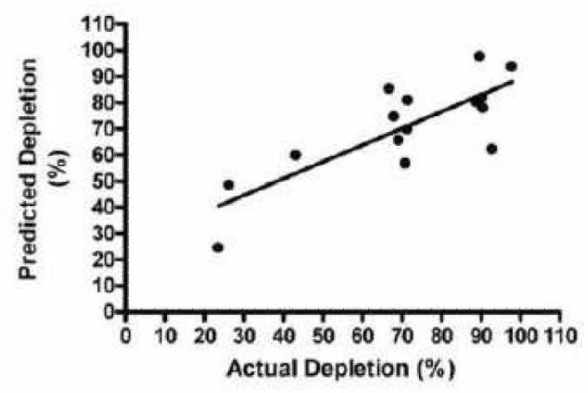

Figure 3.

"Predicted" DA depletion plotted as a function of actual DA depletion for (A) 6-month-old and (B) 18-month-old rats. Values for predicted DA depletion were derived by using the coefficients from the regression equations in Table 2B. It is clear that the relationship between predicted and actual DA depletion is stronger for the middle-aged group than for the young group. 


\section{Table 1}

Striatal DA and DOPAC content (ng/g wet weight +/- SEM) as a function of hemisphere (ipsi=lesioned, contra=non-lesioned), age (6 months vs. 18 months), and time point ( 2 weeks vs. 6 weeks).

\begin{tabular}{lcccc} 
& \multicolumn{2}{c}{ 2 weeks } & \multicolumn{2}{c}{ 6 weeks } \\
\cline { 2 - 5 } & Contra & Ipsi & Contra & Ipsi \\
\hline 6 months & \multicolumn{2}{c}{$\mathrm{n}=9$} & \multicolumn{2}{c}{$\mathrm{n}=8$} \\
\hline DA & $11948 \pm 825$ & $2119 \pm 561$ & $9921 \pm 1211$ & $1903 \pm 360$ \\
DOPAC & $5328 \pm 1358$ & $1865 \pm 566$ & $4840 \pm 532$ & $1683 \pm 262$ \\
\hline 18 months & & & \multicolumn{2}{c}{$\mathrm{n}=8$} \\
\hline DA & $10062 \pm 2476$ & $3872 \pm 1624$ & $12515 \pm 2941$ & $4376 \pm 2448$ \\
DOPAC & $3930 \pm 247$ & $2969 \pm 880$ & $4831 \pm 746$ & $1986 \pm 706$ \\
\hline
\end{tabular}


